\title{
Configuração de Processos de Negócio em Organizações Públicas Considerando Informações de Contexto e Requisitos Não-Funcionais
}

\author{
Amora Sukar $^{1}$, Fernanda Alencar $^{1,2}$, Higor Monteiro $^{1}$, Tarcísio Pereira ${ }^{2,3}$ \\ ${ }^{1}$ Universidade de Pernambuco - UPE \\ ${ }^{2}$ Universidade Federal de Pernambuco - UFPE \\ ${ }^{3}$ Instituto Federal do Sertão Pernambucano - IFSERTÃO-PE \\ aca3@ecomp.poli.br, fernandaalenclecomp.poli.br, \\ higor.monteiro@upe.br, tcp@cin.ufpe.br
}

\begin{abstract}
Resumo. Atualmente, o gerenciamento e a modelagem de processos de negócio são práticas bem estabelecidas no setor privado. No entanto, verifica-se que a orientação a processos nas organizações públicas ainda está em seus estágios iniciais. Devido à natureza dos processos de negócio desse setor, normalmente sujeitos a diversas limitações, tais como leis, diretrizes políticas e orçamentos, é fundamental que seus modelos de processos de negócio sejam suficientemente flexíveis para apoiarem mudanças e restrições. Assim, essa pesquisa tem como objetivo apresentar um diagnóstico sobre como são consideradas informações de contexto e a influência dos Requisitos Não-Funcionais durante a configuração dos processos de negócio no setor público. Para isso, uma Revisão Sistemática da Literatura foi realizada com objetivo de identificar e avaliar abordagens existentes que tratem essas informações em conjunto na modelagem de processos. Também foram realizadas entrevistas em organizações públicas, para analisar como aqueles conceitos são ou podem ser considerados de fato na prática. Ao fim da pesquisa, buscou-se apontar evidências sobre a necessidade de adaptação da modelagem dos processos de negócio atuais para a construção de modelos que sejam sensíveis ao contexto, capazes de considerar Requisitos Não-Funcionais em sua configuração, bem como as vantagens e desvantagens envolvidas nesse processo.
\end{abstract}

Palavras-Chave: Business Process Modeling. Business Process Configuration. Non-Functional Requirements. Context-Awareness. Public Organizations.

\section{Introdução}

Os processos de negócio modernos geralmente operam em ambientes dinâmicos, abertos e não determinísticos. Isso significa que o contexto de execução é volátil e o resultado de algumas atividades não é completamente controlável. Além disso, o conjunto de políticas de negócios também muda dinamicamente. Mudanças no contexto ou re- 
sultados indesejáveis de algumas atividades podem muitas vezes causar o término anormal do processo e impedir a realização dos objetivos de negócio. As empresas precisam lidar, portanto, com a alta variabilidade, bem como com uma forte demanda por flexibilidade dos seus processos [12]. Os processos de negócio (BP) devem ser, portanto, projetados para responder a diferentes eventos e sua especificidade, bem como a condições imprevisíveis em um ambiente. A literatura refere-se a esses processos como processos sensíveis ao contexto. Vários trabalhos de pesquisa mostraram os benefícios da consciência de contexto nos sistemas de Gerenciamento de Processos de Negócio (BPM). O contexto é definido como "o mínimo de variáveis contendo todas as informações relevantes que impactam o projeto e a execução de um BP" [8].

A flexibilidade dos modelos de processos de negócio pode ser tratada usando modelos configuráveis. Esses tipos de modelo são extensões de linguagens de modelagem de processos de negócio que mantêm informações sobre a variabilidade nos mesmos modelos em que foram desenhados [6]. Em termos de configuração de processos, [6] defende o uso de Requisitos Não-Funcionais (RNFs) como critérios adequados para orientar a configuração. Além disso, as informações sobre o contexto podem ser usadas para eliminar soluções inválidas. Os RNFs, às vezes chamado de requisitos de qualidade, definem as restrições que o processo deve cumprir. Estes representam as características de alto nível a partir das quais os processos são normalmente avaliados. Alguns trabalhos já exploram as possibilidades de integrar modelos de processos de negócio e RNFs, como exemplo em [19], [20], [21]. Em [6], essa integração é feita em tempo de design para ser aplicada como uma estratégia de adaptação.

Devido à natureza dos processos de negócio das organizações públicas, os quais estão sujeitos a diversas limitações, tais como leis, diretrizes políticas e orçamentos, é fundamental que seus modelos de processos de negócio sejam suficientemente flexíveis para apoiarem mudanças e restrições [26]. Atualmente, o gerenciamento e a modelagem de processos de negócio são práticas bem estabelecidas no setor privado. No entanto, verifica-se que a orientação a processos na administração pública ainda está em seus estágios iniciais [2].

Com a intenção de contribuir com a maturação de BPM no setor público, essa pesquisa tem como principal objetivo apresentar um diagnóstico sobre como são consideradas informações de contexto e a influência dos RNFs durante a configuração dos processos de negócio nesse setor. Como objetivos específicos: (1) foi realizada uma Revisão Sistemática da Literatura (RSL) para avaliar abordagens existentes que tratem essas definições conjuntamente com a modelagem de processos; (2) foram feitas entrevistas em organizações públicas para analisar como aqueles conceitos são ou podem ser considerados de fato na prática. Ao fim da pesquisa, buscou-se apontar evidências sobre a necessidade de adaptação da modelagem dos processos de negócio atuais para a construção de modelos sensíveis ao contexto capazes de considerar RNFs em sua configuração, bem como as vantagens e desvantagens envolvidas nesse processo.

O trabalho encontra-se estruturado da seguinte forma: na Seção 2, apresenta-se o referencial teórico de base da pesquisa; na Seção 3, são apresentados os procedimentos metodológicos utilizados; na Seção 4, é apresentado o diagnóstico das evidências levantadas; na Seção 5, são apresentados trabalhos relacionados identificados; e, por fim, na Seção 6, são apresentadas as conclusões e trabalhos futuros. 


\section{Referencial Teórico}

Nessa seção são apresentadas as bases teóricas envolvidas na pesquisa.

\subsection{Business Process Management (BPM)}

Um processo de negócio é um conjunto de atividades realizadas de maneira coordenada dentro de um ambiente organizacional e técnico para atingir uma meta de negócio. Dessa forma, pode ser representado por meio de um modelo que cria uma abstração, fornecendo uma compreensão de como várias atividades são conduzidas [7].

O Gerenciamento de Processos de Negócio (do inglês, Business Process Management, BPM) é uma abordagem gerencial que tem recebido crescente interesse da academia e da indústria na última década [1]. O BPM é um paradigma chave da computação empresarial para aumentar a agilidade nas organizações. Foi considerado uma prioridade máxima para as organizações que tentam sobreviver em mercados altamente competitivos. O BPM se refere ao gerenciamento de todo o ciclo de vida do processo de negócio, que inclui: Projeto (design), análise, implementação, execução e melhoria contínua dos processos de uma organização [1].

Devido à complexidade existente no âmbito dos processos, como mecanismo de auxílio, foi proposta uma notação de fácil compreensão por todos os usuários do negócio, desde os analistas de negócio que criam rabiscos iniciais dos processos, até o desenvolvedor técnico, responsável por implementar a tecnologia que irá executar o processo. A ideia foi de tornar mais clara a organização das atividades que compõe um dado processo e ter-se uma padronização de comunicação da organização. Essa linguagem de modelagem de processos de negócio foi então definida pela OMG [5], como a Notação de Modelagem de Processos de Negócio, BPMN (do inglês, Business Process Modeling and Notation).

A maioria dos métodos existentes para o projeto de processos de negócio segue princípios imperativos, ou seja, a ordem dos eventos é predefinida. Como resultado, todos os eventos de processo significativos precisam ser determinados e as ações correspondentes precisam ser predefinidas no tempo de design. No tempo de execução, os processos seguem o modelo configurado com possibilidades limitadas para desviar do cenário predefinido [10]. A definição de modelos de processos de negócio adaptativos e sensíveis ao contexto é de grande interesse. No entanto, as soluções propostas para essa área são limitadas. De fato, a maioria das abordagens existentes que tratam da adaptação dos processos de negócio não leva em conta o contexto. Em vez disso, eles se concentram nos meios intrínsecos de alterar o modelo de processo e suas instâncias depois que uma necessidade de mudança é observada. Essas abordagens levam em conta apenas a parte reativa da adaptação e ignoram os estímulos para a mudança, ou seja, o contexto. No entanto, acredita-se que levar em conta esses estímulos e seu impacto no comportamento do processo é essencial para garantir sua adaptação [16]. 


\subsection{Configuração de Processos de Negócio e Sensibilidade ao Contexto}

A configuração dos processos permite instanciar um modelo de processo de acordo com o contexto e melhorar sua adaptabilidade. Na execução, no entanto, o processo seguirá o modelo pré-configurado ou selecionará de um conjunto limitado de variações [6]. Em um cenário de processos de negócio, em que as organizações devem detectar e responder rapidamente às mudanças em suas rotinas, o contexto é o conjunto mínimo de variáveis que contém todas as informações relevantes que afetam o desenho e a implementação de um processo de negócio [7]. [11] conclui que contexto é qualquer informação implícita e explícita sobre circunstâncias ou situações que afetam uma entidade.

Devido à relevância desse conceito em vários domínios de conhecimento ou aplicações, alguns esforços foram feitos para criar linguagens e meta-modelos que são capazes de especificá-lo [7]. A modelagem de contexto permite a descrição e a estruturação da informação contextual. Vários modelos estão disponíveis para diferentes domínios. [16] afirma que é essencial que as abordagens de engenharia de processos de negócio tenham um modelo para a representação de suas informações contextuais. Há várias classificações que foram propostas para modelos de contexto baseados na estrutura de dados usada para a descrição e adaptações [16]:

- Modelos de valor-chave: O contexto é representado como um par (atributo, valor);

- Modelos baseados em XML: Utilizam uma estrutura de dados hierárquica. A profundidade dessa estrutura depende do contexto descrito;

- Modelos baseados em MDA (Model Driven Architecture): Essa abordagem de modelagem é usada para criar modelos de alto nível que estejam em conformidade com o MOF (Meta Object Facility);

- Modelos gráficos: É uma linguagem para descrever gráficos direcionados rotulados;

- Modelos lógicos: O contexto é expresso como um conjunto de fatos ou regras;

- Modelos baseados em ontologias: Permite especificar conceitos e relações entre diferentes componentes da ontologia.

\subsection{Requisitos Não-Funcionais na Modelagem de Processos de Negócio}

Os Requisitos Não-Funcionais (RNFs) são geralmente associados a atributos de qualidade [19], requisitos extra-funcionais e requisitos não-comportamentais. Eles expressam o comportamento e as restrições que o software deve alcançar [20] [12]. Acessibilidade, segurança, desempenho, portabilidade, consistência, manutenibilidade e eficácia, são alguns exemplos de RNFs. Estes são usados principalmente na engenharia de 
software, embora esse conceito seja adaptável a outros tipos de produtos [21], podendo inclusive ser utilizados como critério para escolha entre alternativas no projeto e implementação de um sistema [22]. Erros e omissões nos RNFs podem levar a um impacto negativo sobre a qualidade do produto final [12].

A importância do Requisitos Não-Funcionais para modelos de processos de negócio tem sido reconhecida nos últimos anos [12]. No BPM, Requisitos Funcionais representam o que o processo de negócio deve executar enquanto os RNFs representam restrições de qualidade que precisam ser atendidas durante a execução do processo [18]. Estes são aplicados no desenho de modelos de processos de negócio por meio de extensões de linguagens de modelagem de processos, permitindo sua análise de forma mais completa. Exemplos típicos de RNFs na engenharia de software também podem ser usados em BPM, como os relacionados a desempenho e usabilidade, por exemplo.

Esses requisitos são comumente declarados tardia e informalmente durante o desenvolvimento do produto, dificultando sua aplicação [21]. Essa fraqueza é evidente no BPM pelo fato de que notações de modelagem de processos de negócio, tal como o BPMN, não apoiarem os RNFs [23]. Além disso, algumas técnicas que foram propostas para representá-los nos processos de negócio não abrangem totalmente o problema. Tais técnicas geralmente levam em consideração apenas como representar os RNFs, sem abordar a fonte de informação necessária para modelá-los [7].

Considerando a necessidade de alinhamento estratégico, um processo de negócio deve ser implementado e executado de acordo com os objetivos estratégicos vinculados ao escopo que abrange esse processo. Esse alinhamento pode ser apoiado por RNFs especificados no modelo de processo de negócio, que é usado para orientar a implementação do processo [18]. Assim, um objetivo estratégico seria representado em um modelo de processo via RNFs [18], além de restrições legais, econômicas e de interoperabilidade [12], que por sua vez seria usado para apoiar a sua implementação de fato.

\section{Procedimentos Metodológicos}

Inicialmente, foi realizada uma Revisão Sistemática da Literatura (RSL) para identificar abordagens que tratem de informações de contexto e requisitos não-funcionais na modelagem de processos de negócio. A princípio, pesquisou-se trabalhos relacionados com o setor público especificamente. No entanto, como não houve resultados satisfatórios, a pesquisa foi ampliada para abordagens em um âmbito geral. Dessa forma, houve uma extensão da RSL realizada por [3], que compreendeu trabalhos publicados dos anos de 2003 a 2012, acrescentando-se à pesquisa trabalhos até o ano de 2018 que considerem esses conceitos.

Posteriormente, foi desenvolvido um protocolo de entrevista semi-estruturada para ser aplicado em organizações públicas. Como afirmado em [1], esse tipo de estratégia de coleta de dados permite um conjunto flexível de perguntas e, durante a entrevista, é possível explorar tópicos emergentes de interesse. Os questionamentos realizados foram baseados nas questões de pesquisa descritas na seção 4 . O objetivo principal foi o de avaliar se ocorrem a elicitação e priorização de Requisitos Não-Funcionais, e a forma como são tratadas no que se refere aos processos de negócio. Além disso, buscou-se 
verificar se informações de contexto são analisadas durante o desenho dos processos na empresa, e como estas afetam a sua configuração. Os entrevistados são servidores públicos responsáveis por promover e/ou executar projetos de BPM. Em resumo, três profissionais de duas instituições foram entrevistados de forma individual, e atualmente executam o papel de analista de processos. Todas as entrevistas foram gravadas em áudio e transcritas separadamente.

\subsection{Condução da RSL}

As strings de busca foram definidas de acordo com as perguntas de pesquisa realizadas. A lista completa das strings de busca e suas combinações são apresentadas na Tabela 1. Essas strings foram aplicadas de acordo com o formato exigido por cada engenho de busca utilizado (IEEE Xplore, Scopus e Engineering Village), filtrando os anos de 2013 a 2018, uma vez que os anos anteriores foram contemplados em [3]. Como resultado da aplicação da estratégia de busca, 377 estudos foram coletados. Após excluir os repetidos, restaram 208 estudos.

Tabela 1. Strings de pesquisa do protocolo de busca da RSL

\begin{tabular}{|c|c|}
\hline Strings & Questões \\
\hline $\begin{array}{l}\text { (1) (non-functional requirements OR NFR OR quality requirements OR quality } \\
\text { attributes) AND (business process OR business process modeling OR BPM) }\end{array}$ & Q1 \\
\hline $\begin{array}{l}\text { (2) (context-aware) AND (business process OR business process modeling OR } \\
\text { BPM) }\end{array}$ & Q2 \\
\hline
\end{tabular}

A partir disso, os artigos foram excluídos de acordo com os critérios de exclusão: estudos que não discutem os conceitos especificados; estudos disponíveis apenas como resumos; trabalhos incompletos e estudos duplicados.

Primeiramente, os critérios de exclusão foram aplicados ao título e abstract, resultando em 67 estudos. Um último filtro ainda foi realizado, analisando a introdução e conclusão, que resultou ao final em 20 estudos. Os resultados obtidos são discutidos na próxima seção.

\section{Diagnóstico}

Nessa seção são apresentadas e analisadas as evidências obtidas a partir dos procedimentos metodológicos utilizados.

\subsection{Análise dos Resultados da RSL}

Nessa seção, são apresentadas as questões de pesquisa e os seus resultados, destacando as evidências recolhidas a partir da catalogação e avaliação dos dados. 


\section{Q1 - Requisitos não-funcionais são representados na modelagem de processos de negócio?}

[19] traz uma abordagem que faz uso de softgoal interdependency graphs (SIGs) para representar RNFs. Símbolos SIG personalizados são usados para vincular os diagramas BPMN e SIG. Usando SIGs, os RNFs são decompostos e, em seguida, conectados às atividades do processo de negócio. Nessa abordagem, os vários RNFs relacionados ao processo desenvolvido são listados para que, com a contribuição das partes interessadas, possam ser classificados de acordo com suas prioridades. Através da conexão do RNF, já decomposto, à atividade no processo de negócio, é possível identificar o fator "make or break" do projeto e dar mais a atenção nessa parte crítica, resultando em melhores chances de um processo bem-sucedido.

[20] propõe uma abordagem que cria uma extensão do Goal Model para derivar um modelo de processo de negócio quality-aware. Combina elementos para expressar os requisitos de qualidade em modelos $i^{*}$ e demonstra a derivação de um modelo consistente de processos de negócio considerando informações de qualidade. Suporta a modelagem de requisitos de qualidade associados aos conceitos do Goal Model com os conceitos correspondentes em processos de negócio, atribuindo requisitos de qualidade a hard-goals, tarefas, recursos e atores. O algoritmo de transformação cria um modelo BPMN com requisitos de qualidade integrados. Para que seja possível definir um intervalo aceitável de qualidade usando métricas, esses requisitos são modelados como anotações e não como softgoals. As anotações estão incluídas no modelo de objetivos e, através do algoritmo proposto, este modelo é transformado em um BPMN que contém características de qualidade para atividades, recursos, objetos de informação e atores. Os requisitos de qualidade baseados nessas características podem ser visualizados nos processos de negócio usando pequenos símbolos. Para cada característica, é dado um símbolo gráfico individual, que é adicionado ao elemento do modelo de processo correspondente.

[21] Propõe uma extensão do meta-modelo BPMN para cobrir os requisitos nãofuncionais. Faz uso de Text Annotations para expressar os RNFs e os relaciona aos elementos do BPMN. Em [22], para modelar os requisitos de qualidade (QRs), a abordagem proposta cria uma extensão para Diagrama de Atividades da UML com dois elementos adicionais: QRProcess (QP) e QRActivityNode (QAN), acrescentando ao modelo um raciocínio quantitativo e qualitativo. Para o raciocínio qualitativo, a abordagem constrói um modelo de QRs combinando o Gráfico de Interdependência Softgoal (SIG) e o Gráfico de Interdependência de Problemas.

Em [23] é apresentado o "MOdel-driven QUality Attributes for business processes" (MOQUA), que visa fornecer uma maneira sistemática de tratar atributos de qualidade por todo o ciclo de vida de BPM. O MOQUA propõe um processo de engenharia orientada ao modelo, no qual os modelos de processos de negócio abstratos e seus atributos de qualidade são refinados sequencialmente por meio de transformações de modelo até a produção de processos de negócio executáveis. Os RNF são identificados na modelagem de negócios de alto nível, como entidades de primeira classe no desenvolvimento de software. Usa a GRL para representar atributos de qualidade dos processos de negócio. A GRL suporta uma modelagem orientada por objetivos, especialmente 
sobre atributos de qualidade. Como o BPMN não fornece recursos de modelagem diretos para analisar alternativas de processo em termos de atributos de qualidade de alto nível ou metas de negócios (soft), uma linguagem de modelagem orientada por objetivos serve como uma boa alternativa para atender a essas necessidades.

Por fim, [6] propõe uma abordagem chamada BVCCoN [48], que visa a configuração de processos de negócio utilizando requisitos não-funcionais e informações contextuais. A abordagem é composta pelas atividades: elicitar variabilidade; descrever variabilidade; analisar contexto; ligar RNFs e variantes; e realizar configuração. As primeiras quatro etapas são realizadas em tempo de design, enquanto que a última etapa é realizada em tempo de execução. Em [4] é desenvolvida uma ferramenta de modelagem que cobre as seguintes atividades dessa abordagem: descrever variabilidade, analisar contexto e ligar RNF e variantes.

Após a análise desses trabalhos, é possível concluir que "sim", os requisitos nãofuncionais estão sendo representados na modelagem de processos de negócio. Como convencionalmente as notações de modelagem cobrem apenas características funcionais do processo, pesquisadores estão propondo novas abordagens, estendendo abordagens já existentes e também encontrando diferentes maneiras de representar os RNFs.

\section{Q2 - Informações de contexto são consideradas na modelagem de processos de ne- gócio?}

[7] Propõe um metamodelo que integra contexto ao processo. Trata de uma abordagem para representar o contexto de uma atividade de um processo de negócio em um determinado domínio. O metamodelo conceitual apoia o processo de adaptação, não considerando apenas as circunstâncias atuais, mas captando também como essas condições afetam o processo.

Nessa proposta, o modelo de processo, derivado do Metamodelo do Processo de $\mathrm{Ne}$ gócio, foi construído com base nos conceitos dessa camada. O Modelo de Contexto foi criado com base nos conceitos do Metamodelo de Contexto, que inclui os elementos Situação e Elemento Contextual. Posteriormente, suas instâncias também foram criadas, o que consequentemente levou à incorporação de outros elementos como Atividade, Papel e Ator.

[14] introduz uma definição formal de um domínio de planejamento que possa lidar com o contexto não estático em todo o modelo de processo. Para abordar a questão da construção de modelos de processos sensíveis ao contexto de forma automatizada, apresenta um algoritmo de planejamento. A fim de realizar a abstração a partir de uma execução de processo individual e construir modelos inteiros de processo, considera estados de crença em vez de estados do mundo. Tem como objetivo construir um modelo de processo abrangente, que não precise ser reconfigurado ou adaptado durante o tempo de execução para considerar o contexto não estático.

[13] traz o conceito de knowledge-intensive process, que são processos que exigem maior esforço na tomada de decisões por parte dos atores para trabalhar de forma eficaz. Dessa forma, quase nenhuma regra de execução de processos de negócio é definida de antemão: atividades são tipicamente definidas em tempo de execução pelos atores do processo após a conclusão da atividade anterior e análise de seus resultados com base 
na situação específica (ou contexto) e experiência. Para aplicar a abordagem, propõese que o processo de negócio seja enriquecido com informações semânticas, convertendo os textos em linguagem natural para as atividades semanticamente anotadas da instância do processo, em vez de descrever o modelo de processo completo de forma estruturada. As principais características mensuráveis do processo devem ser então identificadas para atender às necessidades do cliente. Quando as medidas de tais características ultrapassam certos limites definidos, ações são tomadas para corrigir a situação, enviando sinais para o proprietário do processo identificado.

[12] propõe uma linguagem chamada CAptLang para modelar processos de negócio adaptáveis sensíveis ao contexto. A execução de instâncias do processo de negócio do CAptLang resulta em um conjunto de variantes de processo adaptadas instanciadas no mesmo modelo de processo, mas dinamicamente reestruturadas para lidar com contextos específicos. A evolução do processo explora as informações sobre as variantes do processo para identificar as adaptações recorrentes de melhor desempenho e adotá-las como soluções gerais no modelo de processo. No entanto, as variantes de processo são estritamente relacionadas a contextos de execução específicos e não podem ser adotadas como soluções gerais. Adiciona aos conceitos clássicos da linguagem de fluxo de trabalho (tais como entrada, saída, atividades de manipulação de dados) a possibilidade de relacionar a execução do processo ao contexto do sistema anotando atividades com pré-condições, pós-condições e compensações. Além disso, introduz a possibilidade de especificar atividades abstratas nos processos. Uma atividade abstrata é definida em tempo de design em termos do objetivo que precisa alcançar, expressa como configurações de contexto a serem alcançadas, e é automaticamente refinada no tempo de execução em um processo executável considerando a configuração do contexto atual, com o objetivo de alcançar mecanismos precisos de adaptação.

[11] introduz um framework que ajuda a obter uma visão geral dos fatores contextuais que influenciam o desempenho dos processos. Como primeiro passo, todos os fatores contextuais são classificados pelo critério de origem: dentro ou fora da empresa. $\mathrm{O}$ próximo passo é o de fornecer subgrupos abrangentes para as dimensões interna e externa. De uma perspectiva interna, derivam-se três tipos de contexto: condições organizacionais, recursos e clientes. Todos esses devem ser avaliados para indicar sua relevância. O primeiro aspecto de avaliação é se o fator contextual tem impacto no desempenho. O segundo trata sobre o quanto o contexto é influenciável, e o terceiro sobre o seu grau de dinamismo.

[10] apresenta uma visão do gerenciamento de processos de negócio com base no contexto através de uma modelagem totalmente declarativa combinada com um gerenciamento de contexto inovador e análise de conceito formal. Em vez de considerar o paradigma imperativo tradicional na especificação de processos, são explorados princípios declarativos: um processo de negócio é representado como uma máquina de estados finitos (FSM) - um sistema de transição de estados - que permite lidar com eventos de processo e eventos de contexto que possuem ordem indeterminada de ocorrência, definindo os cenários correspondentes em tempo de execução.

[15] propõe abordagem para facilitar a modelagem de variantes de processo em tempo de design e permitir a adaptação automatizada e controlada de processos em tempo de execução. A entidade central da abordagem é a família de processos sensíveis 
ao contexto (CPF). Em detalhe, um CPF compreende um modelo de processo base com áreas de extensão, situações contextuais baseadas em parâmetros de processo, um conjunto de fragmentos de processo injetados nas áreas de extensão em tempo de execução e um conjunto de especificação de injeção. As áreas de extensão representam a parte dinâmica do processo. Portanto, um modelador de processos pode se concentrar em modelar atividades previsíveis para adicionar as partes dinâmicas do modelo de processo base posteriormente. Em particular, as áreas de extensão são usadas para injetar automaticamente fragmentos de processo no processo de base em tempo de execução com base na situação contextual atual, bem como em especificações de injeção bem definidas.

[17] introduz uma abordagem de como os modelos de processo possíveis são construídos de acordo com diferentes guias em diferentes contextos. Consiste em três etapas: abstrair dependências lógicas e temporais do modelo de processo imperativo; identificar o grupo congelado que são partes essenciais do negócio; identificar as áreas flexíveis; e verificar todos os potenciais modelos de processos colaborativos. O objetivo da abordagem é fornecer visibilidade do processo para diferentes situações de negócios.

Em [9], a abordagem se concentra em, a partir de modelos de metas dos requisitos de negócio, explorar o domínio do negócio em busca das informações de contexto que podem ser percebidas e utilizadas para melhorar a realização de metas de negócios em nível operacional. As informações de contexto consideradas suficientes para justificar seus custos (e outras possíveis desvantagens) são selecionadas para uso na implementação do processo de negócio. Com base na dinâmica das informações de contexto selecionadas, a abordagem ajuda a identificar restrições no posicionamento dos elementos de processo responsáveis por detectar e utilizar essas informações.

A contribuição de [8] consiste no desenho e implementação de uma ferramenta para a seleção dos dados do contexto de execução. A ferramenta analisa o modelo de processo de negócio para selecionar as variáveis contextuais que podem impactar a execução do processo. Para cada atividade projetada, a ferramenta fornece ao usuário a possibilidade de adicionar dados contextuais. Durante a fase de execução, para cada instância do processo, a ferramenta obtém os dados físicos, que correspondem à implementação dos dados de negócios e dos dados contextuais. Primeiro, ele obtém a atividade sendo executada e, depois, obtém as restrições contextuais para produzir a solicitação apropriada para obter o valor dos dados contextuais.

[25] propõe um método original de identificação de alterações, que resolve vários desafios da modelagem de processos com reconhecimento de contexto, através da anotação de modelos e artefatos. Já [24] apresenta um modelo de contexto baseado em ontologia, suporta a semântica declarativa e permite o raciocínio formal em informações contextuais. São extraídas condições de contexto do modelo de contexto como expressões booleanas. Portanto, se uma condição de contexto for atingida, uma adaptação é acionada para responder aos eventos que surgem. Consequentemente, cada condição de contexto é representada como uma tripla (sujeito, predicado, objeto). Descreve a estrutura para BPM com reconhecimento de contexto com base na Linha de Produtos de Software Dinâmico, usando modelos de variabilidade em tempo de execução para suportar a adaptação dinâmica de processos de negócio em resposta a eventos de contexto. 
Em [16], a abordagem proposta é baseada em ontologias e visa identificar e formalizar o conhecimento contextual relevante para os processos de negócio, a fim de ser capaz de adaptar os processos de negócio de acordo com o contexto. Essa abordagem tem a particularidade de ser genérica e extensível, podendo ser integrada a muitas abordagens de modelagem de processos de negócio. Introduz o CM4BPM: um metamodelo para a representação do contexto.

A partir desses trabalhos, conclui-se que informações de contexto estão sendo consideradas na modelagem de processos de negócio. Pesquisadores estão propondo ou adaptando abordagens de processos de negócio já existentes para incluir informações que representem os diferentes tipos de contexto.

\section{2 $\quad$ Entrevistas}

Entrevistas foram conduzidas em organizações públicas e incluíram questionamentos sobre elicitação e priorização de Requisitos Não-Funcionais e sensibilidade ao contexto para processos de negócio existentes na empresa, e como estas afetam a sua configuração. Os entrevistados são servidores públicos responsáveis por promover e/ou executar projetos de BPM. Em resumo, três profissionais de duas instituições foram entrevistados, os quais executam o papel de analista de processos. Todos possuem pós graduação, com no mínimo 6 anos de experiência na área de BPM.

Os entrevistados trabalham nos processos da sua empresa fazendo uso da notação BPMN 2.0 para a modelagem, e da ferramenta Bizagi para o desenho dos modelos. Todos afirmaram que realizam elicitação dos requisitos não-funcionais para se construir os modelos, mas apenas um afirmou que esses requisitos possuem algum tipo de representação na modelagem. Esta, quando realizada, é exibida através dos elementos de anotações de texto. Os demais afirmaram que essa informação é registrada na documentação do processo ou na descrição das atividades. A priorização dos RNFs para o desenho dos modelos é considerada apenas por um deles.

As mudanças de contexto e seus impactos nos processos são reconhecidas por todos os entrevistados, e algumas delas são discutidas nas reuniões que antecedem a modelagem dos processos. No entanto, são representadas nos modelos de forma estática, através de desvios de fluxo ou descritas na documentação. Caso ocorra alguma modificação de contexto imprevista que impacte a estrutura do processo em si, tal como modificação de leis, restrições orçamentárias ou mudanças nas diretrizes organizacionais, esta é redesenhada ou o processo para até que o contexto necessário para sua retomada seja novamente ativado, ou ainda, é realizado de forma $a d-h o c$ através de consulta aos especialistas.

De forma geral, foi observado que nenhuma abordagem padrão é seguida para tratar requisitos não-funcionais, nem tampouco para tratar o fluxo dos processos de acordo com a priorização desses requisitos. $\mathrm{O}$ conceito de mudanças de contexto é reconhecido por todos, no entanto, não são representadas de forma dinâmica através de variabilidade ou configuração de processos. 


\section{Trabalhos Relacionados}

A RSL tratada nesse trabalho foi uma extensão da revisão realizada por [3], no qual os autores apresentam uma análise sistemática visando buscar trabalhos sobre abordagens ou frameworks utilizados para associar RNFs e informações de contexto à modelagem de processos de negócio. No entanto, a busca foi feita limitando-se ao ano de 2012. Para avaliar abordagens mais recentes e sua adequação ao ambiente público, foi necessária a atualização dessa RSL, considerando trabalhos publicados de 2013 até o ano de 2018. A busca realizada pelos autores capturou 24 trabalhos, os quais 14 tratam de RNFs na modelagem de processos de negócio, 9 tratam de informações de contexto, e 1 trata de ambos os conceitos.

Outro trabalho semelhante é realizado em [18], o qual apresenta os resultados de um estudo de literatura conduzido para identificar e avaliar abordagens que propõem o uso de metas estratégicas como suporte para modelagem de RNFs de processos de negócio. A busca foi limitada ao intervalo de 2004 a 2016. Foram identificados 19 trabalhos que abordam objetivos estratégicos, processos de negócio e RNFs. As técnicas mais utilizadas são: i * e Key Perfomance Indicators (KPI), para modelagem de objetivos estratégicos; e Business Process Model and Notation (BPMN), para modelagem de processos de negócio. De acordo com a análise realizada, embora soluções parciais tenham sido encontradas, nenhum trabalho aborda integralmente os RNFs dos processos de negócio com base em objetivos estratégicos. Apesar de haver diversos trabalhos sendo realizados nessa área, foi observado que nenhum deles tratou de forma específica características particulares ao setor público, ou houve qualquer aplicação das abordagens propostas dentro desse setor.

\section{Conclusão e Trabalhos Futuros}

Devido às empresas estarem inseridas em ambientes onde mudanças são constantes, elas precisam manter seus processos atualizados e funcionando adequadamente. Os processos de negócio devem ser, portanto, projetados para responder a diferentes eventos e sua especificidade, bem como a condições imprevisíveis em um ambiente. Nas organizações públicas, a realidade não é diferente. Seus processos, de maneira geral, estão sujeitos a diversas limitações e necessitam ser suficientemente flexíveis para apoiarem mudanças no ambiente e suas restrições.

Atualmente, diversas abordagens têm sido propostas na literatura de forma a considerar nos modelos de processos de negócio restrições de qualidade (RNFs) e informações para representar os diferentes tipos de contexto. Isso permite que os processos possam se adaptar seguindo alguma estratégia, e continuarem funcionando perfeitamente nas condições que foram impostas. Não foi observada, no entanto, qualquer aplicação dessas abordagens no âmbito do setor público.

A partir de entrevistas com servidores públicos, a falta de uso de uma abordagem padrão que considere esses conceitos também foi observada. Com isso, apesar de variações de contexto e RNFs serem reconhecidos durante o trabalho de modelagem de processos, seus impactos na estrutura dos processos não são representados nos modelos. 
Isso pode levar à necessidade de redesenho dos processos, ou mesmo à sua interrupção sem atingir o objetivo proposto.

Como trabalhos futuros, pretende-se ampliar a RSL realizada considerando outros idiomas além do inglês afim de que mais trabalhos possam ser avaliados, e realizar uma análise mais crítica dos resultados alcançados, buscando comparar as abordagens encontradas e avaliar qual delas poderia ser utilizada como base para adaptação de processos pertencentes a uma organização pública. Após isso, vantagens e desvantagens deverão ser levantadas, bem como os ajustes necessários para que seja possível construir, nessas organizações, modelos capazes de se configurar através da sensibilidade ao contexto e dos RNFs.

\section{Agradecimentos}

À Universidade de Pernambuco (UPE) pelos recursos viabilizados para a elaboração desse trabalho.

\section{Referências}

1. Santana, A.F.L., Alves, C.F., Santos, H.R.M., Felix, A.D.L.C.: BPM governance: an exploratory study in public organizations. In: Enterprise, Business-Process and Information Systems Modeling, pp. 46-60. Springer, Berlin, Heidelberg (2011).

2. Ahrend, N., Walser, K., Leopold, H.: Case Study of the Implementation of Business Process Management in Public Administration in Germany, Switzerland and Austria. In: ECEG2013-13th European Conference on eGovernment: ECEG (2013).

3. Pereira, T.C., Alencar, F.M., Silva, J., Castro, J.: Requisitos não-funcionais em modelos de processos de negócio: Uma revisão sistemática. In: IX Simpósio Brasileiro de Sistemas de Informação, pp.37-48 (2013).

4. PEREIRA, T.C.: BVCCoN-Tool: uma ferramenta para apoiar uma abordagem de configuração de processos de negócio dinâmicos. Master's thesis, Universidade Federal de Pernambuco, Recife, Pernambuco, Brasil (2014).

5. OMG Documents Associated With Business Process Model And Notation (BPMN) Version 2.0, http://www.omg.org/spec/BPMN/2.0/, último acesso em 11/2013.

6. Santos, E., Pimentel, J., Pereira, T., Oliveira, K., Castro, J.: Business Process Configuration with NFRs and Context-Awereness. In: ER@BR (2013).

7. da Cunha Mattos, T., Santoro, F.M., Revoredo, K., Nunes, V.T.: A formal representation for context-aware business processes. Computers in Industry 65(8), 1193-1214 (2014).

8. Masse, P.A., Laga, N., Simonin, J.: A Contextual Data Selection Tool for an Enhanced Business Process Analysis. In: e-Business Engineering (ICEBE), pp. 1-8. IEEE (2016).

9. Lapouchnian, A., Yu, E.: Exploring Context Sensing in the Goal-Driven Design of Business Processes. In: Business Informatics (CBI), 18th Conference, 2016, IEEE, Vol. 1, pp. 45-54. IEEE (2016).

10. Rychkova, I., Kirsch-Pinheiro, M., Le Grand, B.: Context-aware agile business process engine: Foundations and architecture. In: Enterprise, Business-Process and Information Systems Modeling, pp. 32-47. Springer, Berlin, Heidelberg (2013). 
11. Kronsbein, D., Meiser, D., Leyer, M.: Conceptualisation of contextual factors for business process performance. In: Proceedings of the International MultiConference of Engineers and Computer Scientists, Vol. 2, pp. 12-14 (2014).

12. Bucchiarone, A., Mezzina, C., Pistore, M.: CAptLang: a language for context-aware and adaptable business processes. In: Proceedings of the Seventh International Workshop on Variability Modelling of Software-intensive Systems, p. 12. ACM (2013).

13. Gromoff, A., Bilinkis, Y., Kazantsev, N.: Business Architecture Flexibility as a Result of Knowledge-Intensive Process Management. Global Journal of Flexible Systems Management 18(1), 73-86 (2017).

14. Heinrich, B., Schön, D.:Automated planning of context-aware process models (2015).

15. Mundbrod, N., Grambow, G., Kolb, J., Reichert, M.: Context-Aware Process Injection. In: OTM Confederated International Conferences "On the Move to Meaningful Internet Systems", pp. 127-145. Springer, Cham (2015).

16. Saidani, O., Rolland, C., Nurcan, S.: Towards a generic context model for BPM. In: 48th Annual Hawaii International Conference on System Sciences (2015).

17. Sofela, O., Xu, L., De Vrieze, P.: Context-Aware Process Modelling through Imperative and Declarative Approach. In: Working Conference on Virtual Enterprises, pp. 191-200. Springer, Berlin, Heidelberg (2013).

18. Carmo, A., Fantinato, M., Thom, L., Prado, E., Spinola, M., Hung, P.: An Analysis of Strategic Goals and Non-Functional Requirements in Business Process Management.

19. Macasaet, R.J., Noguera, M., Rodríguez, M.L., Garrido, J.L., Supakkul, S., Chung, L.: A requirements-based approach for representing micro-business patterns. In: Research Challenges in Information Science (RCIS), 2013 IEEE Seventh International Conference, pp. 112. IEEE (2013).

20. Betz, S., Hickl, S., Oberweis, A.: AAISP: An Approach for Aligning Information Systems Perspectives. In: Software Engineering and Advanced Applications (SEAA), 2016 42th Euromicro Conference, pp. 339-346. IEEE (2016).

21. Bouain, A., El Fazziki, A., Sadgal, M.: Integration of non-functional requirements in a service-oriented and model-driven approach. In: Research Challenges in Information Science (RCIS), 2014 IEEE Eighth International Conference, pp. 1-8. IEEE (2014).

22. Sun, J., Zhao, L., Loucopoulos, P., Zhou, B.: Qra: A quality requirements analysis approach for service systems. In: Services Computing (SCC), 2013 IEEE International Conference, pp. 25-32. IEEE (2013).

23. Teles, F.D.S., Lins, F.A.A., Rosa, N.S.: Realizing quality attributes of service-based business processes: a model-driven approach. In: Computer and Information Technology (CIT), 2014 IEEE International Conference, pp. 450-457. IEEE (2014).

24. Khiari, B., Jilani, L.L.: Towards a DSPL for Context Aware BPM. In: Computer Systems and Applications (AICCSA), 2017 IEEE/ACS 14th International Conference, pp. 12-18. IEEE (2017).

25. Bilinkis, J., Zueva, A., Zaytseva, E.: Context-aware enterprise modelling towards agile models development. In: Future Internet of Things and Cloud Workshops (FiCloudW), 2017 th International Conference, pp. 79-87. IEEE (2017)

26. Jurisch, M.C., Ikas, C., Palka, W., Wolf, P., Krcmar, H.: A review of success factors and challenges of public sector BPR implementations. In: System Science (HICSS), 2012 45th Hawaii International Conference, pp. 2603-2612. IEEE (2012). 